\title{
Improvement of Electromagnetic Compatibility of Motor Drives Using Chaotic PWM
}

\author{
Zheng Wang, K. T. Chau, and Chunhua Liu
}

\author{
Department of Electrical and Electronic Engineering, The University of Hong Kong, Hong Kong, China
}

In this paper, a new chaotic pulse width modulation (PWM) scheme is proposed and implemented to reduce the conducted electromagnetic interference (EMI) in motor drives. The key is the use of logistic mapping to chaoize a frequency-modulated signal which then modulates the carrier frequency. Compared with the sinusoidal PWM and random PWM, the proposed scheme not only suppresses the peaky EMI, but also avoids the occurrence of low-order noises and mechanical resonance. Both simulation and experimental results are given to support the validity.

Index Terms-Chaos, EMC, EMI, motor drives, PWM.

\section{INTRODUCTION}

$\mathbf{I}$ $\mathrm{N}$ the field of variable-speed motor drives, there is a trend for pushing up switching frequencies of pulse width modulation (PWM) inverters to reduce both volume and weight. This trend inevitably contributes to an increasing level of electromagnetic interference (EMI). It leads to degrade the electromagnetic compatibility (EMC) for electronic devices [1]. Consequently, EMC legislation is increasingly stringent in many countries.

In recent years, random PWM (RPWM) schemes, including both nonoptimized and optimized ones, have been developed to improve the EMC of motor drives [2]. The RPWM can effectively spread the discrete spectral power over a continuous spectrum by varying the widths of individual switching intervals randomly. The optimized version can further even out the spectral power distribution. However, these schemes have ignored the consideration of low-order harmonic frequencies and the system natural frequency. So, they inevitably introduce loworder noises, and increase the possibility to create mechanical resonance [3].

Because of the deterministic but random-like behavior, chaos is becoming attractive for application to motor drives [4]. Recently, it has been identified that the use of chaotic PWM to replace sinusoidal PWM (SPWM) can reduce EMI in motor drives [5]. This chaotic PWM scheme employs the Bernoulli shift map to chaoize an amplitude-modulated signal which then modulates the carrier frequency, so-called the chaotically amplitude-modulated PWM (CAMPWM). Although the CAMPWM takes the advantage of easier implementation than the RPWM which needs a truly random source, their performance comparison is absent. Also, the CAMPWM is inflexible to tune the spectral power distribution, and is limited to those chaotic maps satisfying some specific characteristics, namely the mixing rate and probability density function.

In this paper, a chaotically frequency-modulated PWM (CFMPWM) scheme is proposed and implemented, which not only suppresses the peaky EMI, but also avoids the occurrence of low-order noises and mechanical resonance. Both simulation and experimental results will be given to compare the power spectra resulting from SPWM, CFMPWM, and RPWM. More-

Digital Object Identifier 10.1109/TMAG.2007.893712

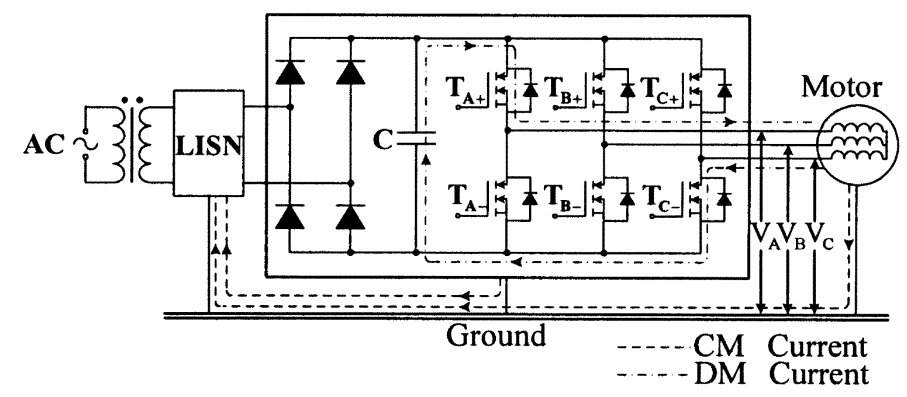

Fig. 1. Conducted EMI in motor drives.

over, a comparison between CFMPWM and CAMPWM will be given and discussed.

\section{PROPOSED CFMPWM}

In general, the total conducted EMI is caused by two mechanisms: the common-mode $(\mathrm{CM})$ noise $V_{\mathrm{CM}}$ which is related to capacitive coupling of switching voltage into the line impedance stabilizing network (LISN), and the differential-mode (DM) noise $V_{\mathrm{DM}}$ which is related to switching current. Fig. 1 shows typical current flows of the conducted EMI in motor drives.

The key of the CFMPWM is to modulate the switching frequency by chaotic sequences. The corresponding carrier signal is given by

$$
\begin{aligned}
V_{C} & =\frac{2}{\pi} \arcsin (X) \\
X & =\sin \left\{\int_{-\infty}^{t}\left[2 \pi f_{\mathrm{sw}}+2 \pi \Delta f \sin \left(\int_{-\infty}^{t} 2 \pi \xi_{i} f_{m} d t\right)\right] d t\right\}
\end{aligned}
$$

where $f_{\mathrm{sw}}$ is the nominal switching frequency, $\Delta f$ is the deviation frequency, $f_{m}$ is the modulation frequency, and $\xi_{i} \in(0,1)$ is the chaotic sequences generated by the logistic map $\xi_{i+1}=$ $A \xi_{i}\left(1-\xi_{i}\right)$ with $A \in[0.1,3.9]$. Hence, the spectrum of $V_{C}$ can be expressed as

$$
S_{n}(f)=\sum_{m=-\infty}^{+\infty} A_{n, m}(\beta) \delta\left(f-n f_{\mathrm{sw}}-m \xi_{i} f_{m}\right)
$$

where $\beta=\Delta f / f_{m}$ is the frequency modulation index, $n$ is the harmonic order, $m$ is the Fourier order, $\delta(\cdot)$ is the im- 


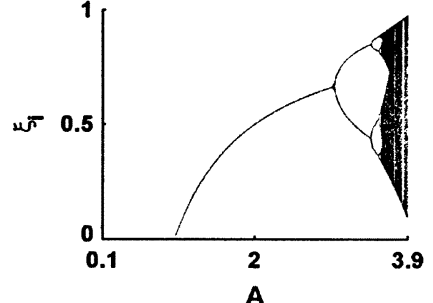

(a)

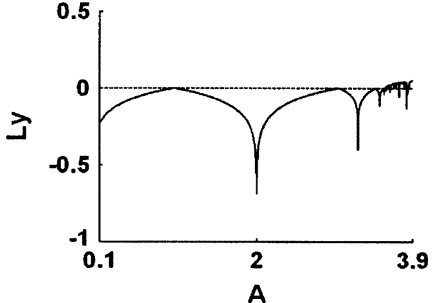

(b)
Fig. 2. Logistic map: (a) Bifurcation diagram; (b) Lyapunov exponent.

pulse function, and $A_{n, m}(\beta)$ is the magnitude coefficient. According to the Carson's rule, if $\beta \gg 1,98 \%$ of the power around $n f_{\mathrm{sw}}$ is distributed to the discrete terms at $f=n f_{\mathrm{sw}}+m \xi_{i} f_{m}$ within a limited frequency range $\left(n f_{\mathrm{sw}}-n \Delta f, n f_{\mathrm{sw}}+n \Delta f\right)$. Fig. 2(a) shows the bifurcation diagram of $\xi_{i}$ against $A$. It illustrates that $A$ can be used to tune the spectral power distribution among no frequency modulation (FM), periodic FM, multi-periodic FM and chaotic FM. When $A \in[3.57,3.9]$, chaos occurs and the corresponding Lyapunov exponent is positive as shown in Fig. 2(b).

Based on a practical 3-phase 400-W 220-V 4-pole induction motor drive, the mechanical resonant frequency of the rotor can be estimated by using the Holzer method. Given $f_{\text {ref }}=30 \mathrm{~Hz}$ and $f_{\mathrm{sw}}=7.5 \mathrm{kHz}$, it deduces $\Delta f=2.2 \mathrm{kHz}$ and $f_{m}=50$ $\mathrm{Hz}$ so as to avoid overlapping with the mechanical resonant frequency of $10.2 \mathrm{kHz}$. Hence, three indicators are defined to assess the EMC of this motor drive. Since the conducted EMI with a frequency exceeding $9 \mathrm{kHz}$ is stringently limited by the VDE standards, the maximum power spectral density (PSD) of $V_{\mathrm{CM}}$ and $V_{\mathrm{DM}}$ within $9-150 \mathrm{kHz}$ is used as the first indicator. In order to evaluate the content of low-order noises while the power spectrum is mainly from $\left(f_{\mathrm{sw}}-\Delta f\right)$ to $\left(f_{\mathrm{sw}}+\Delta f\right)$, the power of $V_{\mathrm{CM}}$ and $V_{\mathrm{DM}}$ within $4.99-5.01 \mathrm{kHz}$ is used as the second indicator. In order to evaluate the possibility of mechanical resonance at $10.2 \mathrm{kHz}$, the power of $V_{\mathrm{CM}}$ and $V_{\mathrm{DM}}$ within $10.19-10.21 \mathrm{kHz}$ is used as the third indicator. Therefore, the CFMPWM is created by tuning $A$ equal to 3.9 so that the first indicator is minimum while the others are low.

\section{Simulation RESULTS}

Computer simulation of the whole system is carried out by using the Matlab Simulink. The corresponding power spectra are plotted by a Matlab function, namely the periodogram method. The power spectra of $V_{\mathrm{CM}}$ and $V_{\mathrm{DM}}$ using SPWM, CFMPWM, and RPWM are computed from zero to $150 \mathrm{kHz}$ under the same $f_{\mathrm{sw}}$ and $\Delta f$. Figs. 3-5 show the corresponding power spectra within $0-22.5 \mathrm{kHz}$ so as to depict their spectral power distributions.

First, by comparing the maximum PSD of their $V_{\mathrm{CM}}$ spectra within $9-150 \mathrm{kHz}$, namely $21.3 \mathrm{dBm} / \mathrm{Hz}$ for SPWM, $10.8 \mathrm{dBm} / \mathrm{Hz}$ for CFMPWM and $7.3 \mathrm{dBm} / \mathrm{Hz}$ for RPWM, it shows that the CFMPWM and RPWM have remarkable improvements over the SPWM. Similarly, by comparing those $V_{\mathrm{DM}}$ spectra, namely $31.4 \mathrm{dBm} / \mathrm{Hz}$ for SPWM, $10.2 \mathrm{dBm} / \mathrm{Hz}$ for CFMPWM, and $6.2 \mathrm{dBm} / \mathrm{Hz}$ for RPWM, it further shows that the CFMPWM and RPWM have remarkable improvements over the SPWM. Thus, it indicates that both the CFMPWM

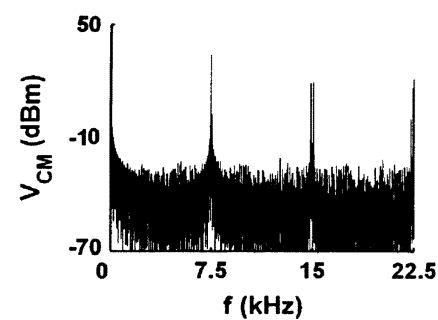

(a)

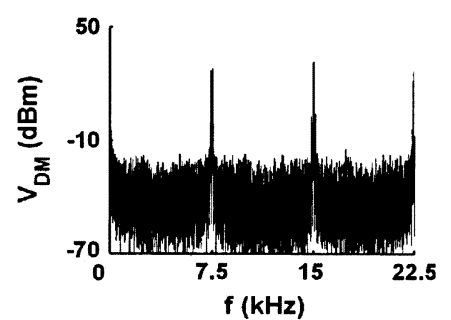

(b)
Fig. 3. Simulated power spectra of SPWM: (a) $V_{\mathrm{CM}}$; (b) $V_{\mathrm{DM}}$.

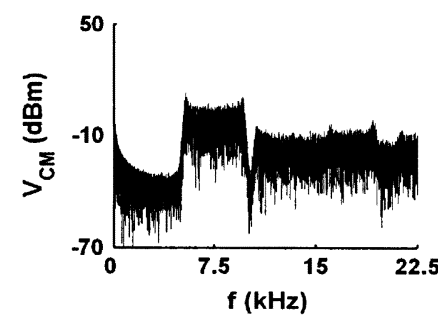

(a)

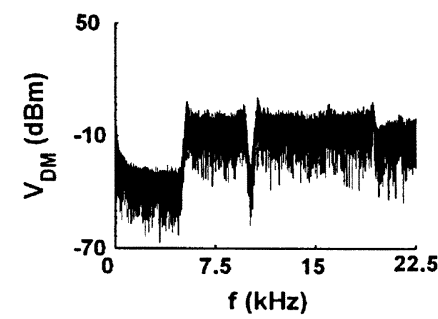

(b)
Fig. 4. Simulated power spectra of CFMPWM: (a) $V_{\mathrm{CM}}$; (b) $V_{\mathrm{DM}}$.

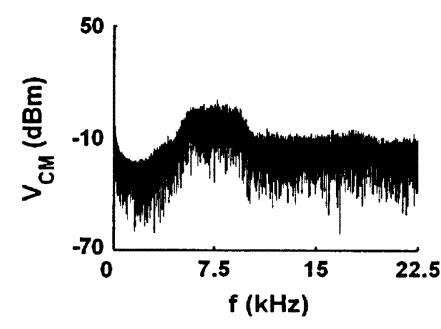

(a)

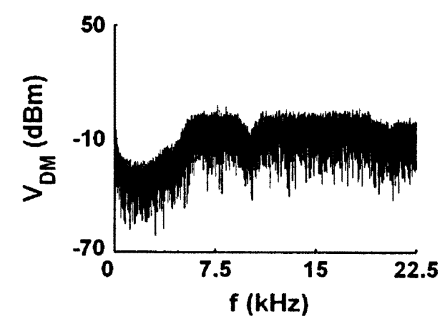

(b)
Fig. 5. Simulated power spectra of RPWM: (a) $V_{\mathrm{CM}}$; (b) $V_{\mathrm{DM}}$.

and RPWM have much better performance than the SPWM in suppressing the peaky EMI.

Second, by comparing the power of their $V_{\mathrm{CM}}$ spectra within $4.99-5.01 \mathrm{kHz}$, namely $-25.8 \mathrm{dBm}$ for SPWM, $-11.4 \mathrm{dBm}$ for CFMPWM, and $2.5 \mathrm{dBm}$ for RPWM, it shows that the CFMPWM has a significant improvement over the RPWM. Similarly, by comparing those $V_{\mathrm{DM}}$ spectra, namely -22.6 $\mathrm{dBm}$ for SPWM, $-13.9 \mathrm{dBm}$ for CFMPWM and $-1.4 \mathrm{dBm}$ for RPWM, it further shows that the CFMPWM has a significant improvement over the RPWM. Thus, this comparison indicates that the CFMPWM has much better performance than RPWM in avoiding low-order noises.

Third, by comparing the power of their $V_{\mathrm{CM}}$ spectra within $10.19-10.21 \mathrm{kHz}$, namely $-25.8 \mathrm{dBm}$ for SPWM, $-21.2 \mathrm{dBm}$ for CFMPWM, and $-0.4 \mathrm{dBm}$ for RPWM, it illustrates that the CFMPWM provides a much greater attenuation than the RPWM. Similarly, by comparing those $V_{\mathrm{DM}}$ spectra, namely $-25.7 \mathrm{dBm}$ for SPWM, $-15.6 \mathrm{dBm}$ for CFMPWM, and -0.2 $\mathrm{dBm}$ for RPWM, it further illustrates that the CFMPWM provides a much greater attenuation than the RPWM. Thus, it indicates that the CFMPWM has much better performance than RPWM in avoiding mechanical resonance.

It should be noted that even though the SPWM offers the lowest spectral power within $4.99-5.01 \mathrm{kHz}$ and $10.19-10.21 \mathrm{kHz}$, its severely peaky EMI will create annoying 


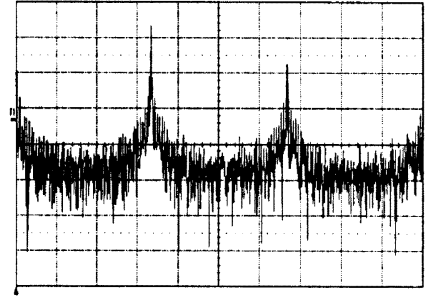

$X: 0-22.5 \mathrm{kHz} ; 2.25 \mathrm{kHz} / \mathrm{div}$ Y: -70 50dBm; 15dBm/div

(a)

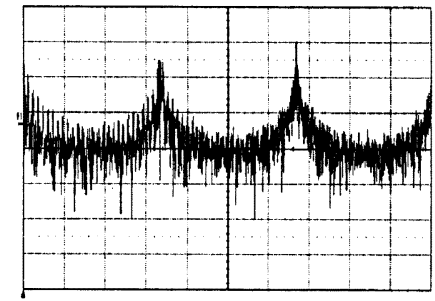

$X: 0 \sim 22.5 \mathrm{kHz} ; 2.25 \mathrm{kHz} / \mathrm{div}$ Y: $-70 \sim 50 \mathrm{dBm} ; 15 \mathrm{dBm} / \mathrm{div}$

(b)
Fig. 6. Measured power spectra of SPWM: (a) $V_{\mathrm{CM}}$; (b) $V_{\mathrm{DM}}$.

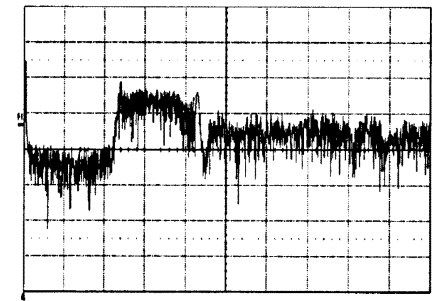
Y: $-70 \sim 50 \mathrm{dBm} ; 15 \mathrm{dBm} / \mathrm{div}$

(a)
$X: 0 \sim 22.5 \mathrm{kHz} ; 2.25 \mathrm{kHz} / \mathrm{div}$

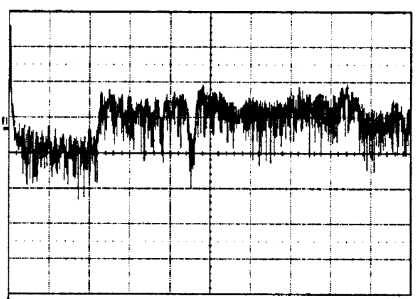

$X: 0 \sim 22.5 \mathrm{kHz} ; 2.25 \mathrm{kHz} / \mathrm{div}$ $Y:-70 \sim 50 \mathrm{dBm} ; 15 \mathrm{dBm} / \mathrm{div}$

(b)
Fig. 7. Measured power spectra of CFMPWM: (a) $V_{\mathrm{CM}}$; (b) $V_{\mathrm{DM}}$.

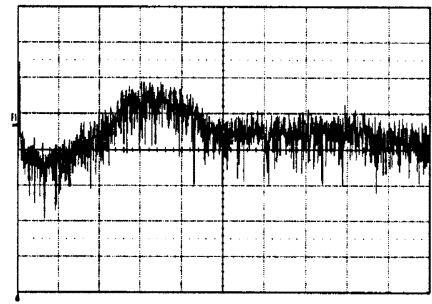

$X: 0 \sim 22.5 \mathrm{kHz} ; 2.25 \mathrm{kHz} / \mathrm{div}$ Y: $-70 \sim 50 \mathrm{dBm} ; 15 \mathrm{dBm} / \mathrm{div}$

(a)

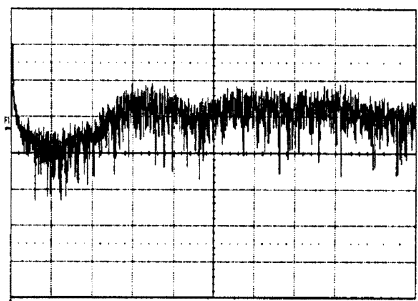

$X: 0 \sim 22.5 \mathrm{kHz} ; 2.25 \mathrm{kHz} / \mathrm{div}$ Y: $-70 \sim 50 \mathrm{dBm} ; 15 \mathrm{dBm} / \mathrm{div}$

(b)
Fig. 8. Measured power spectra of RPWM: (a) $V_{\mathrm{CM}}$; (b) $V_{\mathrm{DM}}$.

acoustic noise in motor drives. This problem precludes the SPWM competing with CFMPWM and RPWM.

\section{EXPERIMENTAL RESULTS}

For experimental verification, an IGBT-based voltage source inverter is constructed to supply the aforementioned induction motor. The CFMPWM scheme is implemented by a single-chip TMS320F240 microcontroller. All power spectra are directly measured by using the LeCroy WR6050 power spectrum analyzer. Figs. 6, 7, and 8 show the measured power spectra of SPWM, CFMPWM, and RPWM, respectively. The resolution bandwidth is set to be $15 \mathrm{~Hz}$. It can be seen that the measured spectra well agree with the simulated counterparts as shown in Figs. 3, 4, and 5. Hence, it experimentally verifies that the proposed CFMPWM not only effectively suppresses the peaky EMI, but also exhibits high immunity to low-order noises and mechanical resonance.

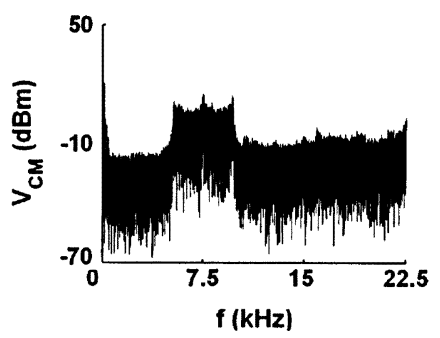

(a)

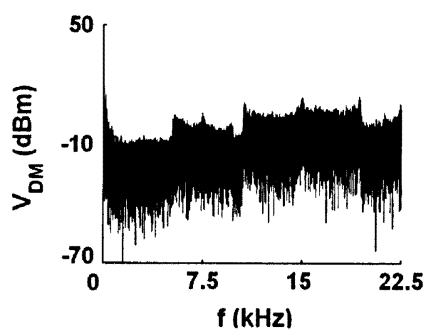

(b)
Fig. 9. Simulated power spectra of CAMPWM: (a) $V_{\mathrm{CM}}$; (b) $V_{\mathrm{DM}}$.

\section{FURTHER COMPARISON}

In order to compare the CFMPWM with the CAMPWM, the power spectra of $V_{\mathrm{CM}}$ and $V_{\mathrm{DM}}$ using CAMPWM are computed under the same logistic map, $f_{\mathrm{sw}}$ and $\Delta f$. Fig. 9 shows their distributions within $0-22.5 \mathrm{kHz}$. The maximum PSD within $9-150 \mathrm{kHz}$, the power within $4.99-5.01 \mathrm{kHz}$, and the power within $10.19-10.21 \mathrm{kHz}$ of $V_{\mathrm{CM}}$ using CAMPWM are $14.4 \mathrm{dBm} / \mathrm{Hz},-4.6 \mathrm{dBm}$, and $-5.6 \mathrm{dBm}$, respectively, whereas those using CFMPWM are only $10.8 \mathrm{dBm} / \mathrm{Hz},-11.4$ $\mathrm{dBm}$, and $-21.2 \mathrm{dBm}$, respectively. Relative data of $V_{\mathrm{DM}}$ are similar. Hence, it confirms that the proposed CFMPWM can offer better spectral performances than the CAMPWM.

\section{CONCLUSION}

By using the logistic map to chaoize the frequency-modulated signal for the carrier frequency, a new CFMPWM has been proposed and implemented to improve EMC of motor drives. Both simulation and experimental results have confirmed that the proposed CFMPWM can effectively suppress the peaky EMI which usually occurs in SPWM, and can avoid the possibility of low-order noises and mechanical resonance which generally occur in RPWM. Moreover, the CFMPWM can offer better spectral performances than the CAMPWM.

\section{ACKNOWLEDGMENT}

This work was supported and funded by a Grant (HKU7154/ 04E) from the Research Grants Council, Hong Kong Special Administrative Region, China.

\section{REFERENCES}

[1] A. M. Sitzia, A. E. Baker, T. W. Preston, A. Puzo, and A. Pons, "Finite element analysis for power electronics EMC applications," IEEE Trans. Magn., vol. 32, no. 3, pp. 1517-1520, May 1996.

[2] F. Blaabjerg, J. K. Pedersen, L. Oestergaard, R. L. Kirlin, A. M. Trzynadlowski, and S. Legowski, "Optimized and non-optimized random modulation techniques for VSI drives," EPE J., vol. 6, pp. 46-53, Sep. 1996.

[3] W. C. Lo, C. C. Chan, Z. Q. Zhu, L. Xu, D. Howe, and K. T. Chau, "Acoustic noise radiated by PWM-controlled induction machine drives," IEEE Trans. Ind. Electron., vol. 47, pp. 880-889, Aug. 2000.

[4] K. T. Chau and Z. Wang, "Design of permanent magnets to chaoize doubly salient permanent magnet motors for electric compaction," $J$. Appl. Phys., vol. 99, pp. 08R306:1-08R306:3, Apr. 2006.

[5] A. Bellini, G. Franceschini, R. Rovatti, G. Setti, and C. Tassoni, "Generation of low-EMI PWM patterns for induction drives with chaotic maps," IEEE IECON, pp. 1527-1532, 2001.

Manuscript received October 31, 2006 (e-mail: zwang@eee.hku.hk). 